\title{
Glutamate excitotoxicity induced by orally administered propionic acid, a short chain fatty acid can be ameliorated by bee pollen

\author{
Afaf El-Ansary ${ }^{1,3,4,6^{*}}$, Huda S. Al-Salem², Alqahtani Asma ${ }^{1}$ and Abeer Al-Dbass ${ }^{5}$
}

\begin{abstract}
Background: Rodent models may guide investigations towards identifying either environmental neuro-toxicants or drugs with neuro-therapeutic effects. This work aims to study the therapeutic effects of bee pollen on brain glutamate excitotoxicity and the impaired glutamine-glutamate- gamma amino butyric acid (GABA) circuit induced by propionic acid (PPA), a short chain fatty acid, in rat pups.

Methods: Twenty-four young male Western Albino rats 3-4 weeks of age, and 45-60 g body weight were enrolled in the present study. They were grouped into four equal groups: Group 1, the control received phosphate buffered saline at the same time of PPA adminstration; Group 2, received $750 \mathrm{mg} / \mathrm{kg}$ body weight divided into 3 equal daily doses and served as acute neurotoxic dose of PPA; Group 3, received 750 mg/kg body weight divided in 10 equal doses of $75 \mathrm{mg} / \mathrm{kg}$ body weight/day, and served as the sub-acute group; and Group 4, the therapeutic group, was treated with bee pollen (50 mg/kg body weight) for 30 days after acute PPA intoxication. GABA, glutamate and glutamine were measured in the brain homogenates of the four groups.
\end{abstract}

Results: The results showed that PPA caused multiple signs of excitotoxicity, as measured by the elevation of glutamate and the glutamate/glutamine ratio and the decrease of GABA, glutamine and the GABA/glutamate ratio. Bee pollen was effective in counteracting the neurotoxic effects of PPA to a certain extent.

Conclusion: In conclusion, bee pollen demonstrates ameliorating effects on glutamate excitotoxicity and the impaired glutamine-glutamate-GABA circuit as two etiological mechanisms in PPA-induced neurotoxicity.

Keywords: Propionic acid, Bee pollen, Glutamate excitotoxicity, Glutamine, GABA

\section{Background}

Autism as a neurodevelopmental disorder is characterized by severe social impairment and communication, repetitive behavior, and restricted interests. Several studies recorded abnormality of glutamatergic signaling pathways in autism. Glutamate receptors are mainly localized in brain areas that have been repeatedly implicated in autism, such as the cerebellum and hippocampus [1] Excitatory glutamate signaling through glutamate receptors (GluRs) plays a key role in cortical development [2] which also indicate its involvement in the etiology of autistic features. Glutamate (Glu) is an amino acid that functions as an

\footnotetext{
* Correspondence: afafkelansary@gmail.com

${ }^{1}$ Central Laboratory, Female Center for Medical Studies and Scientific Section, King Saud University, Riyadh, Saudi Arabia

${ }^{3}$ Autism Research and Treatment Center, Riyadh, Saudi Arabia

Full list of author information is available at the end of the article
}

excitatory neurotransmitter in the central nervous system (CNS). Excitation causes depolarization of the postsynaptic neuron and promotes the propagation of action potentials. In contrast, GABA, the main inhibitory neurotransmitter, results in the hyperpolarization of the postsynaptic cell membrane, dampening the generation of action potentials.

Glutamate is usually synthesized in the neuron and is then transported into glial cells from the synaptic cleft, it is converted into Gln by the enzyme glutamine synthetase (GS), which is completely absent in neuronal cells [3]. Glutamine is then transported back into the neuron, where it is deaminated by glutaminase, once again forming Glu [3].

It is well known that Glu signaling is involved in a wide variety of psychological and cognitive processes. 
Alterations of this signaling have been associated with depression, learning disability and many neurodevelopmental disorders, including autism [4]. A balance between excitatory and inhibitory neurotransmission is critically important, and the loss of this balance is related to autism [5]. Several studies demonstrate the increased levels of plasma and brain Glu together with lower levels of GABA, Gln or their relative ratios to Glu (i.e., Glu/GABA and Glu/Gln) in autistic children, especially those with normal IQ [6]. Moreover, a relationship between Glu excitotoxicity and neuroinflammation in autism was ascertained and suggests that Glu signaling is an attractive target for the development of a therapeutic strategy for autism through Glu receptors and /or transporters, as important proteins playing a critical role in Glu homeostasis [7].

Propionic acid is a metabolite of clostridia species, bacteria found at higher levels in autistic feces compared to controls. Propionic acid was found to be capable of inducing biochemical, behavioral, electrophysiological, and neuropathological changes in rats similar to those observed in autistic patients when given either orally $[8,9]$ or through direct cerebroventricular infusion [10]. In our recently published work, serotonin, dopamine, and noradrenaline as three important neurotransmitters related to autism was remarkably impaired in PPA-treated juvenile rats and were also ameliorated with bee pollen [9]. Additionally, bee pollen demonstrated anti-inflammatory and anti-apoptotic effects through the reduction of PPA induced levels of IFN- $\gamma$ and caspase- 3 as neuroinflammatory and apoptotic markers respectively [9].

Apitherapy is the medical use of honey bee products. This includes the use of honey, pollen, bee bread, propolis, royal jelly, and bee venom. Several studies on neuroinflammatory diseases in animal models have increasingly supported the effectiveness of this therapy in treating diseases related to microglia activation and Glu excitotoxicity among which is autism $[11,12]$. The apitherapeutic mechanism of bee pollen was recently attributed to its antimicrobial activity and potency to induce the regeneration of damaged tissues [13]. It has also been shown that the ethyl alcohol extract of pollen recorded antibiotic activity against Gram-positive pathogenic bacteria, including Klebsiella pneumonia (A propionibacterium) and Pseudomonas aeruginosa and fungi such as Candida albicans. All these microbial organisms, together with Clostridia, are overgrown in most autistic patients, especially those frequently treated with ampicillin as a broad spectrum antibiotic. In addition, bee pollen is known to have detoxification activity, such as heavy metals (Mercury and lead), as a pathological mechanism involved in autism [14]. Pollen also acts through anti-inflammatory mechanisms through the inhibition of the activities of cyclooxygenase and lipoxygenase. These enzymes are responsible for the conversion of arachidonic acid into toxic compounds, such as prostaglandin and leukotrienes, which are inducers of acute and chronic inflammatory conditions and are biomarkers of autism [15].

It is well documented that Glu excitotoxicity is clinically related to neuroinflammation, oxidative stress, and apoptosis $[16,17]$. In our recent study, bee pollen was found to be effective in ameliorating signs of PPAinduced brain toxicity, measured as a depletion of serotonin $(5 \mathrm{HT})$, dopamine and nor-adrenaline as important neurotransmitters related to autism, significant increase of IFN- $\gamma$ and caspase 3 as markers of neuroinflammation and apoptosis respectively [18].

This information motivates our interest to investigate the therapeutic effects of bee pollen on Glu excitotoxicity-related parameters in rat pups orally administered PPA as a rodent model of autism [8]. Glutamate excitotoxicity was selected because it is ascertained as an etiological mechanism in autism directly related to the activation of microglia effectively treated with apitherapy $[10,19]$.

\section{Methods}

The experimental assays for this study were performed on 24 young (3-4 weeks of age) male western albino rats (45 to $60 \mathrm{~g}$ ). Rats used in the present study were bred at the animal house of the pharmacy college, King Saud University, and were randomly assigned to four groups of six rats each. The first group consisted of rats to which only phosphate buffered saline was given at the time the PPA was administered to ensure that treatment administration is matched among comparison groups and was used as a control group. The second and third groups were orally administered a neurotoxic dose of propionic acid (PPA) $(750 \mathrm{mg} /$ $\mathrm{kg}$ body weight divided in 3 doses of $250 \mathrm{mg} / \mathrm{kg}$ body weight/day served as the acute group [8], and $750 \mathrm{mg} / \mathrm{kg}$ body weight divided in 10 equal doses of $75 \mathrm{mg} / \mathrm{kg}$ body weight/day served as the sub-acute group). The fourth group received an oral dose $(50 \mathrm{mg} / \mathrm{kg}$ body weight for 30 days) of 100\% natural bee pollen, imported from Wadi Al-Nahil, a marketing company in Saudi Arabia, after being treated with an acute dose of PPA. The four groups of rats were housed under controlled temperature $\left(21 \pm 1{ }^{\circ} \mathrm{C}\right)$ with ad libitum access to food and water. The animals were sacrificed at the end of each treatment. The protocol of the present work was approved by the Ethics Committee at the King Saud University and all experiments were performed in accordance with the guidelines of the National Animal Care and Use Committee. Figure 1, summarizes the experimental design of the present work.

\section{Tissue preparation}

At the end of the experiment, the rats were anesthetized with carbon dioxide and decapitated. The brain was 


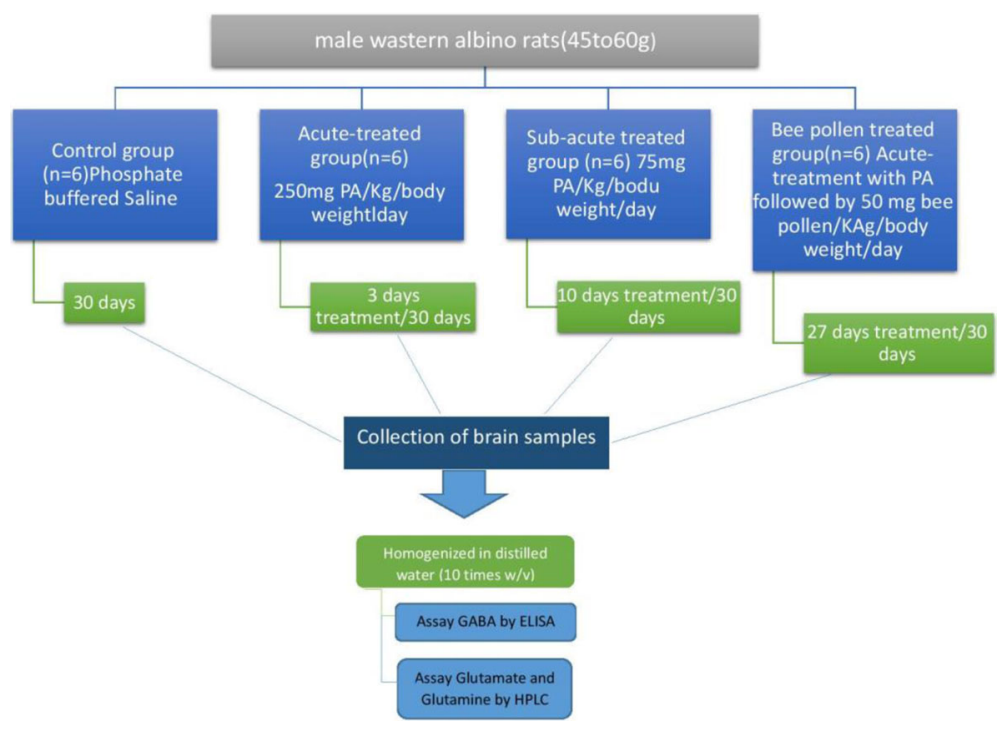

Fig. 1 Schematic presentation of the experimental work demonstrating, dosage, duration of different studied groups

removed from the skull and was dissected into small pieces to be homogenized in distilled water (10 times $w / v)$.

\section{Measurement of GABA levels}

GABA was quantitatively determined using the ELISA immunoassay kit from ALPCO Diagnostics (Salem, NH, USA). Derivatization of extracted GABA was performed using the D-reagent. A quantity of $25 \mu \mathrm{L}$ of the derivatives was then used for subsequent ELISA assays according to the provided instructions. This product has a wide detection limit range of $15-1500 \mathrm{ng} / \mathrm{ml}$.

\section{Measurement of Glu and Gln levels}

Glutamate and Gln levels were assessed using an HPLC method. Brain homogenate samples $(0.1 \mathrm{ml})$ were mixed with $5 \mu \mathrm{l}$ mercaptoethanol and allowed to stand for $5 \mathrm{~min}$ at room temperature, then precipitated with icecold methanol while being vortexed. Tubes were allowed to stand for $15 \mathrm{~min}$ in an ice bucket before the samples were separated by centrifugation (5000 rpm for $15 \mathrm{~min}$ ) and the supernatant was collected. The protein-free supernatants were processed immediately for HPLC analysis of the two amino acids [20].

\section{Statistical analysis}

The data were analyzed using the Statistical Package for the Social Sciences (SPSS, Chicago, IL, USA). The results were expressed as the means \pm S.D. All statistical comparisons between the control and PA and pollentreated rat groups were performed using the one-way analysis of variance (ANOVA) tests with Dunnett's test for multiple comparisons. Significance was assigned at the level of $P<0.05$. Receiver operating characteristics curve (ROC) analysis was performed. The area under the curve (AUC), cutoff values, and the degrees of specificity and sensitivity were calculated. Pearson's correlations were performed between the measured parameters.

\section{Results}

Results are presented as the means \pm S.D. and the percentage change of at least six independent measurements. Table 1 and Fig. 2 present the mean \pm S.D. of the absolute values of Glu, GABA, and Gln together with the two relative values of GABA/Glu and Glu/Gln in the brain homogenates of the four studied groups of rats. Compared to the control groups, the PPA-treated rats exhibited a significant increase in Glu and the Glu/Gln ratio with a concomitant decrease of GABA, Gln, and the GABA/Glu ratio. Figure 3 demonstrates the percentage change of the measured parameters relative to the control group. Glutamate showed $111.56 \%$ and $46.47 \%$ increases with acute and sub-acute PPA treatments, respectively. The decrease in GABA was more pronounced in sub-acute compared to acute samples, recording $64.74 \%$ and $52.46 \%$, respectively. Glutamine was also much lower in sub-acute PPA-treated rat pups, recording a $13.42 \%$ decrease compared to a value of only $7.67 \%$ in acute-treated rats. The recorded decrease of GABA/ Glu and increase of Glu/Gln ratios were more or less similar in both PPA-treated groups. The ameliorating effect of bee pollen was observed through the remarkable decrease of glutamate together with the increase of GABA and Gln in PPA-acute neuro-intoxicated rats.

Figure 4 shows the Pearson's correlations between the three absolute values of the three measured parameters together with the relative concentrations of GABA/Glu 
Table 1 Mean \pm S.D. of the absolute and relative concentrations of GABA, Glu, and GIn in control, PPA-intoxicated and PPA-intoxicated and pollen-treated rat pups

\begin{tabular}{|c|c|c|c|c|c|c|}
\hline Parameter & Group & $N$ & Mean \pm S.D & $\begin{array}{l}\text { Percent } \\
\text { Change }\end{array}$ & $P$ value $^{a}$ & $P$ value \\
\hline \multirow[t]{4}{*}{ GABA (ng/100 mg) } & Control & 6 & $2.95 \pm 0.24$ & 100.00 & & \\
\hline & PPA-acute & 6 & $1.40 \pm 0.29$ & 47.54 & 0.001 & \\
\hline & PPA-sub-acute & 6 & $1.04 \pm 0.25$ & 35.26 & 0.001 & 0.042 \\
\hline & PPA -acute-Pollen & 6 & $1.70 \pm 0.24$ & 57.67 & 0.001 & 0.079 \\
\hline \multirow[t]{4}{*}{ Glu ( $\mu \mathrm{g} / \mathrm{mg})$} & Control & 6 & $1.44 \pm 0.11$ & 100.00 & & \\
\hline & PPA-acute & 6 & $3.05 \pm 0.27$ & 211.56 & 0.001 & \\
\hline & PPA-sub-acute & 6 & $2.11 \pm 0.18$ & 146.47 & 0.001 & 0.001 \\
\hline & PPA -acute-Pollen & 6 & $1.85 \pm 0.10$ & 128.09 & 0.05 & 0.001 \\
\hline \multirow[t]{4}{*}{ GABA/Glu } & Control & 6 & $2.04 \pm 0.06$ & 100.00 & & \\
\hline & PPA-acute & 6 & $0.46 \pm 0.11$ & 22.67 & 0.001 & \\
\hline & PPA-sub-acute & 6 & $0.50 \pm 0.14$ & 24.30 & 0.001 & 0.658 \\
\hline & PPA -acute-Pollen & 6 & $0.92 \pm 0.11$ & 44.98 & 0.046 & 0.001 \\
\hline \multirow[t]{4}{*}{$\mathrm{Gln}(\mu \mathrm{g} / \mathrm{mg})$} & Control & 6 & $0.70 \pm 0.07$ & 100.00 & & \\
\hline & PPA-acute & 6 & $0.64 \pm 0.08$ & 92.33 & 0.272 & \\
\hline & PPA-sub-acute & 6 & $0.60 \pm 0.14$ & 86.58 & 0.176 & 0.559 \\
\hline & PPA -acute-Pollen & 6 & $0.71 \pm 0.05$ & 101.91 & 0.722 & 0.126 \\
\hline \multirow[t]{4}{*}{ Glue/Gln } & Control & 6 & $2.09 \pm 0.19$ & 100.00 & & \\
\hline & PPA-acute & 6 & $4.80 \pm 0.52$ & 230.02 & 0.001 & \\
\hline & PPA-sub-acute & 6 & $3.65 \pm 0.81$ & 174.83 & 0.004 & 0.015 \\
\hline & PPA -acute-Pollen & 6 & $2.62 \pm 0.30$ & 125.78 & 0.004 & 0.001 \\
\hline
\end{tabular}

${ }^{\mathrm{a}} \mathrm{P}$ value between the control group and other groups

${ }^{\mathrm{b}} \mathrm{P}$ value between the PPA-acute group and other groups

and Glu/Gln. Negative and positive correlations are illustrated.

Table 2 and Fig. 5 demonstrate the AUC, specificity, and sensitivity measured markers in PPA-acute and subacute intoxicated rats together with the pollen-treated group. It was shown that while glutamate and glutamine can be used as markers for PPA-acute neurotoxicity (AUC of almost 1) with $100 \%$ or near $100 \%$ sensitivity and specificity, GABA shows high validity as a marker for sub-acute toxicity (AUC $=0.949$ ), with $100 \%$ sensitivity and $77.8 \%$ specificity. GABA/Glu showed equal predictiveness values for both modes of PPA intoxication with AUCs of 0.852 and 0.815 and satisfactory sensitivity and specificity. However, all the measured parameters demonstrate fair validity as biomarkers for pollen's therapeutic effect (AUC range between 0.6-0.7).

Table 3 demonstrates the multiple regression analysis using Gln as a dependent variable. It can be easily

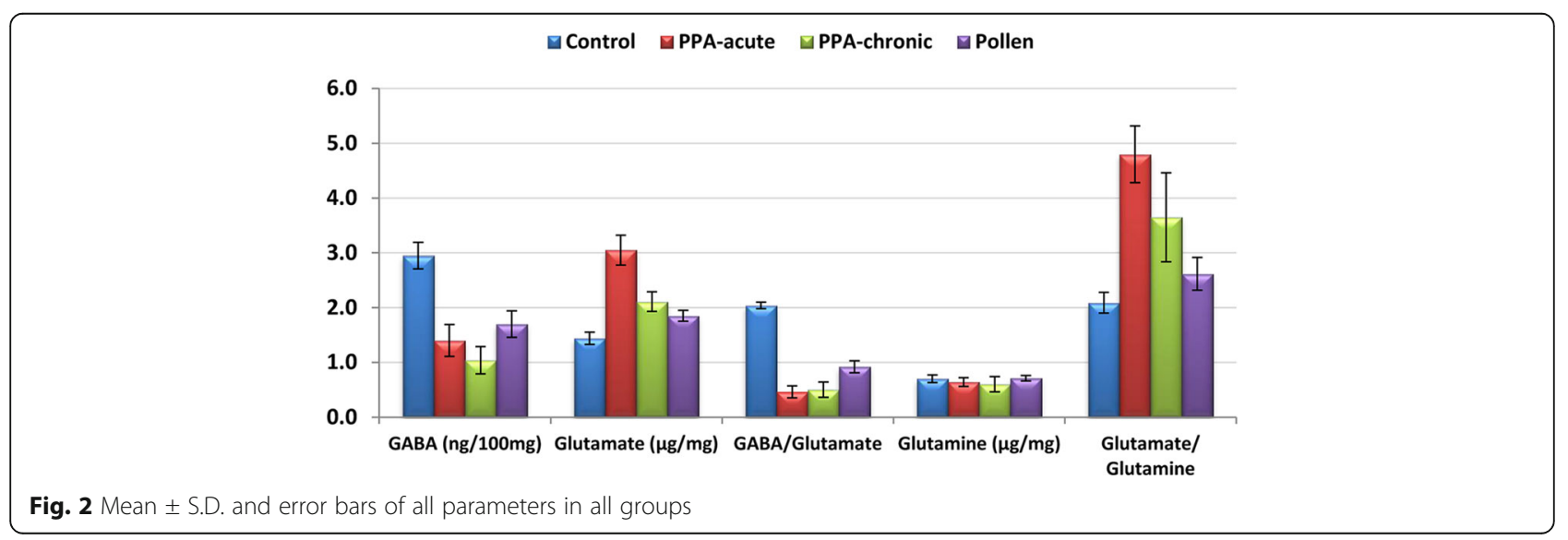




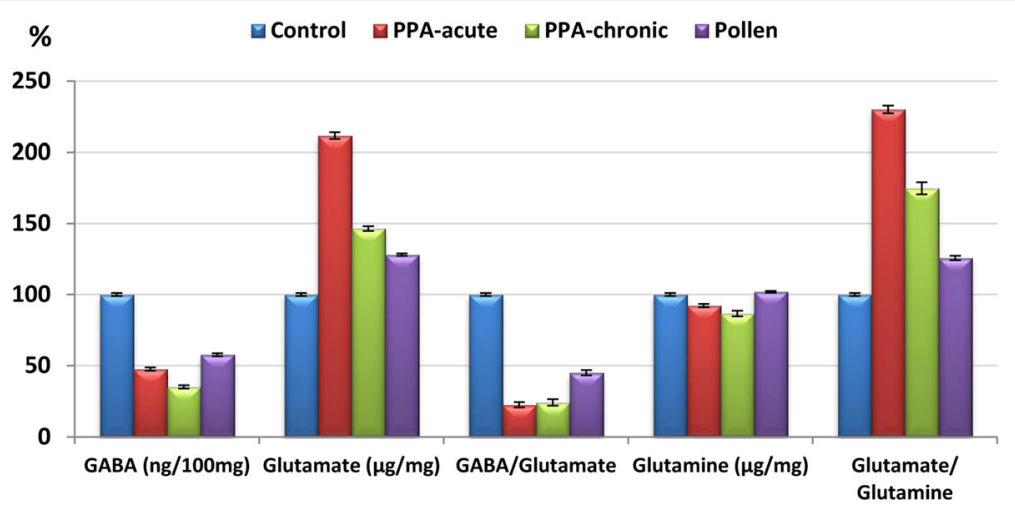

Fig. 3 Percentage increase or decrease of the measured parameters relative to controls, diagrammatically presented as 100\%

observed that the $R^{2}$ value of 0.973 shows that $97.3 \%$ of the increase in Glu is related to an impaired Glu-Gln cycle through which Glu released from the neuron cells is transported to the astrocytes, where it can be converted to Gln.

\section{Discussion}

It is well known that the increase of Glu can activate glutamate receptors, which may mediate excitotoxicity and lead to neuronal cell death. Microglial activation together with the overstimulation of Glu receptors has a principle role in Glu excitotoxicity, which might lead to abnormal brain development and synaptic plasticity and be implicated in PPA neurotoxicity [21].

The present study revealed that a PPA dose of $750 \mathrm{mg} / \mathrm{kg}$ body weight, given either acutely (250 mg/kg body wt. daily for three subsequent days) or sub-acutely given over a 10 days, can induce a significant increase of Glu in the rat brain. It was shown that while sub-acute PPA treatment induced a greater reduction of GABA and Gln as an inhibitory neurotransmitter and Glu buffering product, respectively, acute treatment was more effective in inducing a much higher increase in the excitatory Glu neurotransmitter.
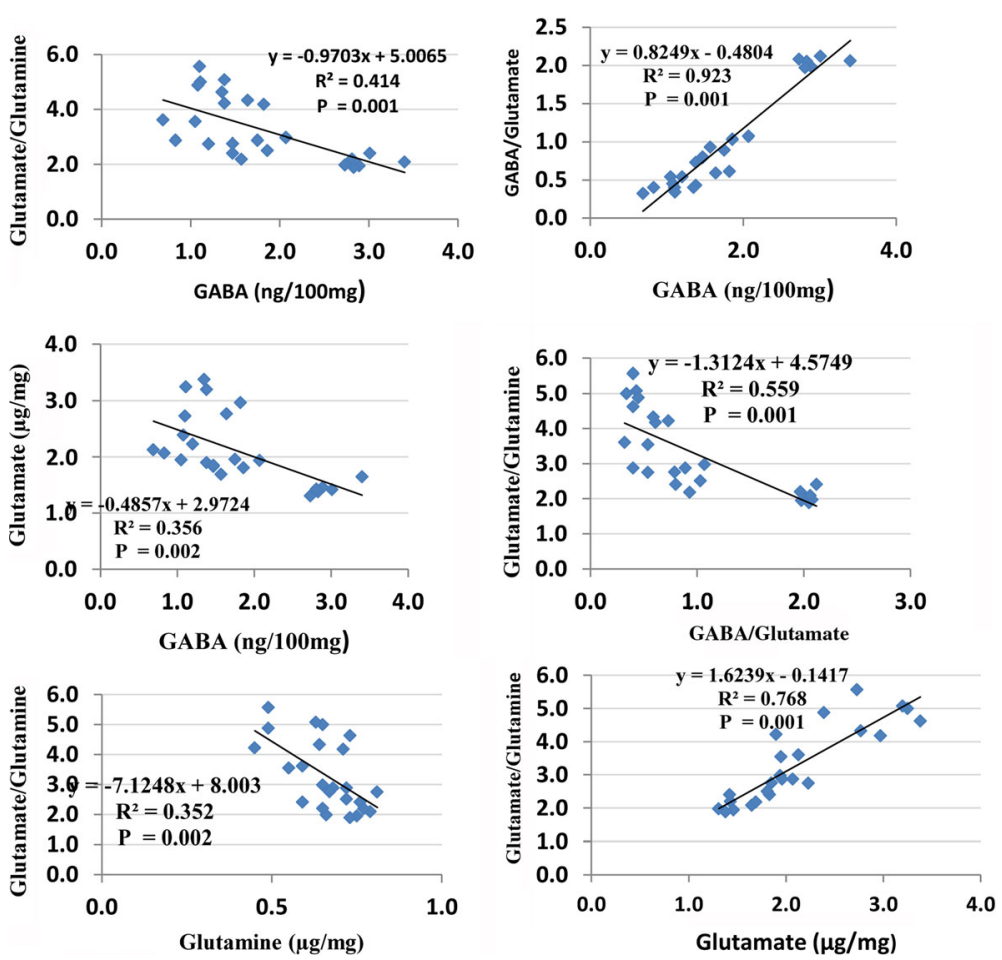

Fig. 4 Positive and negative Pearson's correlations between the measured parameters with a best fit line curve 
Table 2 ROC curve of the measured absolute and relative parameters in all groups

\begin{tabular}{|c|c|c|c|c|c|}
\hline Parameter & Group & Area under the curve & Best Cutoff value & Sensitivity \% & Specificity \% \\
\hline \multirow[t]{3}{*}{ GABA (ng/100 mg) } & PPA-acute & 0.653 & 1.840 & $100.0 \%$ & $44.4 \%$ \\
\hline & PPA-sub-acute & 0.949 & 1.425 & $100.0 \%$ & $77.8 \%$ \\
\hline & Pollen & 0.602 & 1.425 & $100.0 \%$ & $55.6 \%$ \\
\hline \multirow[t]{3}{*}{ Glu ( $\mu \mathrm{g} / \mathrm{mg})$} & PPA-acute & 1.000 & 2.560 & $100.0 \%$ & $100.0 \%$ \\
\hline & PPA- sub-acute & 0.639 & 1.875 & $100.0 \%$ & $55.6 \%$ \\
\hline & Pollen & 0.639 & 2.015 & $100.0 \%$ & $55.6 \%$ \\
\hline \multirow[t]{3}{*}{ GABA/Glu } & PPA-acute & 0.852 & 0.670 & $100.0 \%$ & $72.2 \%$ \\
\hline & PPA- sub-acute & 0.815 & 0.761 & $100.0 \%$ & $66.7 \%$ \\
\hline & Pollen & 0.667 & 0.761 & $100.0 \%$ & $66.7 \%$ \\
\hline \multirow[t]{3}{*}{$\mathrm{Gln}(\mu \mathrm{g} / \mathrm{mg})$} & PPA-acute & 0.630 & 0.655 & $66.7 \%$ & $61.1 \%$ \\
\hline & PPA- sub-acute & 0.699 & 0.610 & $66.7 \%$ & $88.9 \%$ \\
\hline & Pollen & 0.699 & 0.665 & $83.3 \%$ & $61.1 \%$ \\
\hline \multirow[t]{3}{*}{ Glu/Gln } & PPA-acute & 0.963 & 3.897 & $100.0 \%$ & $88.9 \%$ \\
\hline & PPA- sub-acute & 0.657 & 2.634 & $100.0 \%$ & $50.0 \%$ \\
\hline & Pollen & 0.639 & 3.265 & $100.0 \%$ & $55.6 \%$ \\
\hline
\end{tabular}

In the present study, the involvement of Glu in PPAinduced neurotoxicity was examined because Glu is believed to play important roles in various types of neuronal death and its elevation might be due to leakage from dead cells. This can be supported by considering the previous work of El-Ansary et al. $[8,9]$ in which the same dose of PPA $(750 \mathrm{mg} / \mathrm{kg}$ body wt.) was toxic enough to induce a significant increase of caspase 3 as a marker of brain cell death. Moreover, it can find more support in the recent histopathological study which proved that PPA- treatment induced severe cortical neuronal density with ballooning fibrillar acidophilic cytoplasm, degenerated, atrophied, and necrotic neurons together with amygdala mitochondrial crystalysis and microtubular distortion [22]. Both neuron necrosis and mitochondrial crystalysis as histopathological changes can be related to glutamate excitotoxicity. Upon exposure to glutamate, delayed calcium de-regulation usually followed by necrosis, and superoxide anions-induced mitochondrial dysfunction [23, 24].

GABA is derived from glutamate under the decarboxylation action of glutamate decarboxylase (GAD) via GlnGlu-GABA circulation; thus, changes in the Glu level could affect the GABA content as well. Studies have found that a single dose of the severe neurotoxic compound amphetamine-type stimulant (ATS) $(30 \mathrm{mg} / \mathrm{kg})$ in mice down-regulated the Gln/Glu and GABA/Glu ratios, which suggested a circulatory disturbance of the Gln-Glu-GABA circuit [25]. In the present study, PPA demonstrated a level of neurotoxicity comparable to that of ATS [24]. The recorded changes in Glu-Gln -GABA circulation support the persistent autistic features induced in rat pups through the use of orally administered PPA $[8,9]$. It was also related to the results of Tanaka et al. [26], who generated an animal model with behavioral and neuroanatomical abnormalities similar to those observed in autism through the over-stimulation of Glu receptors by the genetic down-regulation of the glial Glu transporters GLT1 and GLAST [26]. This may lead to a decreased uptake of glu from the synaptic cleft and result in excitotoxicity due to a significant elevation of the extracellular Glu level. Moreover, the significantly higher Glu/Gln and lower GABA/Glu ratios presented in Table 1 and Fig. 2 ascertain the effectiveness of PPA in inducing the rodent model of autism.

It is well known that the Glu-Gln cycle in the brain functions to control the levels of glutamate and to shuttle nitrogen between astrocytes and neurons. Under normal physiological conditions, Glu is metabolized in astrocytes by the GS reaction rather than by GAD, which favors Gln formation [26]. The remarkable increase of the Glu:Gln ratio in PPA-treated pups can be considered among the persistent autistic features related to PPA neurotoxicity and to the animal modeling of autism. This is supported by the work of Abu Shmais et al. [20], who recorded a significant increase in the Glu:Gln ratio in autistic patients compared to controls and suggested that the Glu-Gln cycle was greatly affected in these patients.

The significantly lower GABA level and GABA/Glu ratio found in PPA-treated pups could be caused either by a loss of GABAergic interneurons, a decrease in GABA synthesis, or alterations in the astrocytic cycling of GABA, Glu, and Gln [27]. All of these suggested mechanisms have been found as phenotypes in autism and are related to cognitive impairment [27, 28]. 


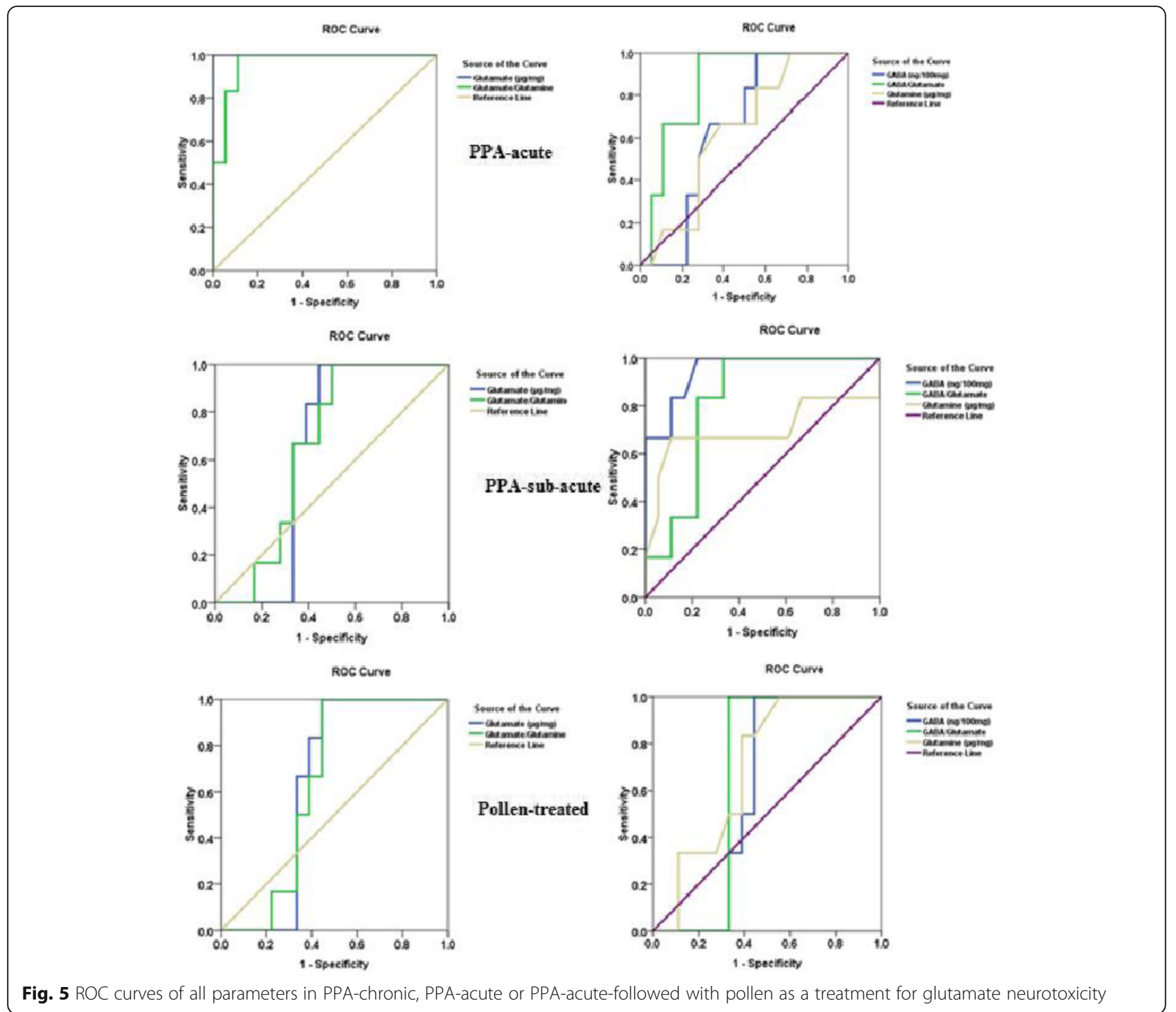

It is well accepted that oxidative stress and the extracellular release of Glu are reciprocally related [29]. Moreover, GS is highly sensitive to ROS-induced damage, which can also impair inhibitory processes, such as GABA-stimulated chloride uptake [29, 30]. Thus, the neurotoxic effect of PPA reported in the current study represented by the impaired Gln-Glu-GABA circuit may be related to its oxidative effect previously reported as the induction of lipid peroxidation, glutathione depletion, decreased catalase and increased glutathione-s-

Table 3 Multiple regression stepwise analysis with glutamate as dependent variable

\begin{tabular}{|c|c|c|c|c|c|}
\hline \multirow[t]{2}{*}{ Predictor Variable } & \multirow[t]{2}{*}{ Beta } & \multirow[t]{2}{*}{$P$ value } & \multirow{2}{*}{$\begin{array}{l}\text { Adjusted } \\
\text { R square }\end{array}$} & \multicolumn{2}{|l|}{ Model } \\
\hline & & & & F value & $P$ value \\
\hline Glutamate/Glutamine & 1.211 & 0.001 & 0.973 & 410.254 & 0.001 \\
\hline Glutamine $(\mu \mathrm{g} / \mathrm{mg})$ & 0.565 & 0.001 & & & \\
\hline
\end{tabular}

transferase, together with the impairment of energy metabolism [6, 7, 31].

Bee pollen has a wide variety of health protection functions due to its many active components, which are mainly composed of sugars, proteins, free amino acids, vitamins, flavonoids, and coenzyme Q10 [29]. As a natural product, pollen is rich with proteins (22.7\%), essential fatty acids (5.1\%), phospholipids (0.45\%), phytosterols (1.5\%), digestible carbohydrates $(30.8 \%)$, reducing sugars (mainly fructose and glucose) (25.7\%), phenolic compounds (1.6\%), and flavonoids (3\%). Pollen is a significant source of vitamins (1.4\%), such as vitamins E, A, D, B complex, folic acid and biotin [32, 33]. Most of these components were individually effective either in improving behavior in valproic acid rat model, or clinically in reducing symptoms of neurodevelopmental disorders among which is autism [33-37]. 
Table 1 and Figs. 2 and 3 demonstrate the ameliorating effect of bee pollen on the Gln-Glu-GABA circuit as a neurotoxic effect of PPA. It was observed that the therapeutic effect of bee pollen is presented as a less significant difference in the measured parameters compared to controls than that recorded by both groups of PPAtreated rat pups. Based on the reciprocal relationship between Glu excitotoxicity and oxidative stress, the therapeutic effect of bee pollen can be attributed to its antioxidant effect. This can be supported by considering the antioxidant and neuroprotective effects of coenzyme Q10 as a component of bee pollen [30]. This suggestion is in good agreement with the recent work of AlGhamdi et al. [38], which demonstrated that coenzyme Q10 has potential protective and restorative effects against PPA-induced brain injury, as confirmed by improvements in biochemical markers and DNA double-strand breaks.

Moreover, the reported therapeutic effect of bee pollen can also be attributed to its content of flavonoids as one of the most potent antioxidant compounds. The amelioration of glutamate toxicity measured as (Glu / Gln) ratio, is consistent with the study of Yang et al. [39] and Kim et al. [40] which ascertain the role of flavonoids as major component of bee pollen in the attenuation of glutamate-induced neurotoxicity. This can find more support through considering the fact that flavonoids and their metabolites have been found in the brain tissues of rodents after oral administration [41], suggesting their ability to cross the blood-brain barrier.

The positive and negative correlations between the measured parameters presented in Fig. 4 ascertain the importance of the Glu-Gln-GABA circuit as a persistent biochemical autistic feature, which can be targeted to ameliorate the neurotoxic effect of PPA.

In receiver operating characteristics (ROC) analysis, while an AUC value close to 1 was an excellent diagnostic and predictive marker, a curve that lay close to the diagonal (AUC $=0.5$ ) had no diagnostic utility. An AUC value close to 1 was always accompanied by satisfactory values of specificity and sensitivity of the biomarker. Table 2 and Fig. 5 demonstrate that among the three measured parameters, while GABA can be used as perfect marker for sub-acute PPA neurotoxicity $($ AUC $=0.949)$, was recorded $($ AUC $=1)$ in cases of acute treatment with PPA. The GABA/Glu ratio recorded more or less the same predictive value for both modes of toxicity. Glutamine was the least predictive recorded AUC value in the range of 0.6-0.7, which showed a relative predictive value for the three treated groups (acute, sub-acute, and pollen-treated). Table 3 also shows that GABA, Glu, GABA/Glu, Gln, and Glu/Gln can be used as fair markers for the ameliorating effect of bee pollen treatment (AUCs 0.6-0.7).

\section{Conclusion}

These findings suggest that bee pollen is an attractive candidate for further studies for the development of new treatment strategies that can target glutamate excitotoxicity and oxidative stress as two etiological mechanisms of autism. Higher doses of bee pollen may yield more ameliorating effects, but because pollen is heavily under-researched it cannot be recommended for any particular usage in autistic patients until more studies are conducted.

\section{Abbreviations \\ AUC: Area under the curve; CNS: Central Nervous System; GABA: Gamma aminobutyric acid; Gln: Glutamine; Glu: Glutamate; GS: Glutamine synthetase; PPA: Propionic acid; ROC: Receiver Operating Characteristics}

\section{Acknowledgement}

This research project was funded by a grant from the research center of the Center for Female Scientific and Medical Colleges at King Saud University.

\section{Competing interests}

The authors declare that they have no competing interests.

\section{Funding}

This work was funded by the research center, Center for Female Scientific and Medical Colleges at King Saud University, Saudi Arabia.

\section{Availability of data and materials}

The data will not be shared because authors intend to build a second research paper based on the currently presented data.

\section{Authors' contributions}

AE: Suggested the use of propionic acid for autism modeling and drafted the manuscript. HA: Suggested the use of bee pollen and revised the manuscript. AQ: Help in the design and improvement of figures. AA: Co-drafted the manuscript. All authors read and approved the final manuscript.

Consent for publication

Not applicable.

\section{Author details}

${ }^{1}$ Central Laboratory, Female Center for Medical Studies and Scientific Section, King Saud University, Riyadh, Saudi Arabia. ${ }^{2}$ Department of Pharmaceutical Chemistry, College of Pharmacy, King Saud University, Riyadh, Saudi Arabia. ${ }^{3}$ Autism Research and Treatment Center, Riyadh, Saudi Arabia. ${ }^{4}$ Shaik AL-Amodi Autism Research Chair, King Saud University, Riyadh, Saudi Arabia. ${ }^{5}$ Department of Biochemistry, Science College, King Saud University, Riyadh, Saudi Arabia. ${ }^{6}$ Medicinal Chemistry Department, National Research Centre, Dokki, Cairo, Egypt.

Received: 25 October 2016 Accepted: 12 May 2017 Published online: 22 May 2017

\section{References}

1. Ozawa S, Kamiya H, Tsuzuki K. Glutamate receptors in the mammalian central nervous system. Prog Neurobiol. 1998;54:581-618.

2. Manent JB, Represa A. Neurotransmitters and brainmaturation: early paracrine actions of GABA and glutamate modulate neuronal migration. Neuroscientist. 2007;13:268-79.

3. El-Ansary A, Al-Ayadhi L. GABAergic/glutamatergic imbalance relative to excessive neuroinflammation in autism spectrum disorders. J Neuroinflammation. 2014;11:189.

4. Wei J, Yao L, Yang L, Zhao W, Shi S, Cai Q, Chen D, Li W, Wang Q. Alteration of glutamate/GABA balance during acute alcohol intoxication in rats: effect of Xingnaojing injection. J Ethnopharmacol. 2015;166:333-9.

5. Rosa SG, Quines CB, Stangherlin EC, Nogueira CW. Diphenyl diselenide ameliorates monosodium glutamate induced anxiety-like behavior in rats by modulating hippocampal BDNF-Akt pathway and uptake of GABA and serotonin neurotransmitters. Physiol Behav. 2016;155:1-8. 
6. Tu WJ, Chen H, He J. Application of LC-MS/MS analysis of plasma amino acids profiles in children with autism. J Clin Biochem Nutr. 2012;51(3):248-9.

7. Ghanizadeh A. Targeting of glycine site on NMDA receptor as a possible new strategy for autism treatment. Neurochem Res. 2012;36(5):922-3.

8. El-Ansary AK, Ben Bacha A, Kotb M. Etiology of autistic features: the persisting neurotoxic effects of propionic acid. J Neuroinflammation. 2012:9:74.

9. Al-Salem HS, Bhat RS, Al-Ayadhi L, El-Ansary A. Therapeutic potency of bee pollen against biochemical autistic features induced through acute and sub-acute neurotoxicity of orally administered propionic acid. BMC Complement Altern Med. 2016;16:120.

10. MacFabe DF, Cain DP, Rodriguez-Capote K, Franklin AE, Hoffman JE, Boond F, Taylor AR, Kavaliers M, Ossenkopp KP. Neurobiological effects of intraventricular propionic acid in rats: possible role of short chain fatty acids on the pathogenesis and characteristics of autism spectrum disorders. Behav Brain Res. 2007:176:149.

11. Han S, Lee K, Yeo J, Kweon H, Woo S, Lee M, Baek H, Kim S, Park K. Effect of honey bee venom on microglial cells nitric oxide and tumor necrosis factoralpha production stimulated by LPS. J Ethnopharmacol. 2007;111(1):176-81.

12. Suzuki K, Sugihara G, Ouchi Y, Nakamura K, Futatsubashi M, Takebayashi K, Yoshihara Y, Omata K, Matsumoto K, Tsuchiya K, Iwata Y, Tsujii M, Sugiyama T, Mori N. Microglial activation in young adults with autism spectrum disorder. JAMA Psychiatry, 2013. 7 0(1):49-58.

13. Komosinska-Vassev K, Olczyk P, Kaźmierczak J, Mencner L, Olczyk K. Bee pollen chemical composition and therapeutic application Evid based. Complement Alternat Med. 2015;297425

14. Eraslan G, Kanbur M, Silici S, Liman B, Altinordulu S, Sarica ZS. Evaluation of protective effect of bee pollen against propoxur toxicity in rat. Ecotoxicol Environ Saf. 2009;72930:931-7.

15. El-Ansary A, Shaker G, Siddiqi NJ, Al-Ayadhi LY. Possible ameliorative effects of antioxidants on propionic acid / clindamycin - induced neurotoxicity in Syrian hamsters. Gut Pathog. 2013;5(1):32.

16. Zeidán-Chuliá F, Salmina AB, Malinovskaya NA, Noda M, Verkhratsky A Moreira JC. The glial perspective of autism spectrum disorders. Neurosci Biobehav Rev. 2014 Jan;38:160-72.

17. Di Marco B, Bonaccorso CM, Aloisi E, D'Antoni S, Catania MV. Neuroinflammatory mechanisms in developmental disorders associated with intellectual disability and autism Spectrum disorder: a Neuro- immune perspective. CNS Neurol Disord Drug Targets. 2016;15(4):448-63.

18. El-Ansary. A Data of multiple regressions analysis between selected biomarkers related to glutamate excitotoxicity and oxidative stress in Saudi autistic patients. Data Brief. 2016 Feb 15;7:111-6.

19. Thomas RH, Foley KA, Mepham JR, Tichenoff LJ, Possmayer F, MacFabe DF. Altered brain phospholipids and acylcarnitine profiles in propionic acid infused rodents: further development of a potential model of autism spectrum disorders. J Neurochem. 2010;113:515-29.

20. Abu Shmais GA, Al-Ayadhi LY, Al-Dbass AM, El-Ansary AK, et al. J Neurodev Disord. 2012:4(1):4

21. Flores-Méndez M, Mendez-Flores OG, Ortega A. Glia plasma membrane transporters: key key players in glutamatergic neurotransmission. Neurochem Int. 2016;98:46-55.

22. Khalil SR, Abd-Elhakim YM YM, Selim ME, Al-Ayadhi LY. Apitoxin protects rat pups brain from propionic acid-induced oxidative stress: the expression pattern of Bcl-2 and Caspase-3 apoptotic genes. Neurotoxicology. 2015;49:121-31.

23. Nicholls DG, Budd SL, Ward MW, Castilho RF. Excitotoxicity and mitochondria. Biochem Soc Symp. 1999;66:55-67.

24. Rueda CB, Llorente-Folch I, Traba J, Amigo I, Gonzalez-Sanchez P, Contreras L, Juaristi I, Martinez-Valero P, Pardo B, Del Arco A, Satrustegui J. Glutamate excitotoxicity and $\mathrm{Ca} 2+$ regulation of respiration: role ofthe $\mathrm{Ca} 2+$ activated mitochondrial transporters (CaMCs). Biochim Biophys Acta. 2016;1857(8): $1158-66$.

25. Tapiero H, Mathé G, Couvreur P, Tew KD. Free amino acids in human health and pathologies II. Glutamine and glutamate. Biomed Pharmacother. 2002; 56:446-57.

26. Tanaka K. Role of glutamate transporters in the pathophysiology of major mental illnesses. Nihon Shinkei Seishin Yakurigaku Zasshi. 2009;29:161.

27. Rae CD. A guide to the metabolic pathways and function of metabolites observed in human brain $1 \mathrm{H}$ magnetic resonance spectra. Neurochem Res. 2014:39:1-36.

28. Gaetz W, Bloy L, Wang DJ, Port RG, Blaskey L, Levy SE, Roberts TP. GABA estimation in the brains of children on the autism spectrum: measurement precision and regional cortical variation. Neurolmage. 2014;86:1-9.
29. Levy DI, Sucher NJ, Lipton SA. Redox mediation of NMDA-mediated toxicity in mammalian central nervous system. Neurosci Lett. 1990;110:291-6.

30. Xue X, Zhao J, Chen L, Zhou J, Yue B, Li Y, Wu L, Liu F. Analysis of coenzyme Q10 in bee pollen using online cleanup by accelerated solvent extraction and high performance liquid chromatography. Food Chem. 2012; 1133(2):573-8

31. MacFabe DF, Rodríguez-Capote $K$, Hoffman JE, Franklin AE, Mohammad-Asef Y, Taylor AR, Boon F, Cain DP, Kavaliers M, Possmayer F, Ossenkopp KP. A novel rodent model of autism: Intraventricular infusions of Propionic acid increase Locomotor activity and induce Neuroinflammation and oxidative stress in discrete regions of adult rat brain. Am J Biochem Biotechnol. 2008; 4(2):146-66

32. Kędzia B, Hołderna-Kędzia E. New studies on biological properties of pollen. In: Postępy Fitoterapii, vol. 1; 2012. p. 48-54.

33. Almeida-Muradian LB, Pamplona LC, Coimbra S, Barth OM. Chemical composition and botanical evaluation of dried bee pollen pellets. J Food Compos Anal. 2005;18(1):105-11.

34. Du L, Zhao G, Duan Z, Li F. Behavioral improvements in a valproic acid rat model of autism following vitamin D supplementation. Psychiatry Res. 2017; 253:28-32.

35. Al-Farsi YM, Waly MI, Deth RC, Al-Sharbati MM, Al-Shafaee M, Al-Farsi O, Al-Khaduri MM, Gupta I, Ali A, Al-Khalili M, Al-Adawi S, Hodgson NW, Ouhtit A. Low folate and vitaminB12 nourishment is common in Omani children with newlydiagnosed autism. Nutrition. 2013;29(3):537-41.

36. Fluegge K. Propionic acid metabolism, ASD, and vitamin B12: is there a role for environmental nitrous oxide? Int J Devl Neurosci. 2017:57:21-3.

37. Gumpricht $\mathrm{E}$, Rockway S. Can $\omega-3$ fatty acids and tocotrienol-rich vitamin $\mathrm{E}$ reduce symptoms of neurodevelopmental disorders? Nutrition. 2014;30(78):733-8.

38. Al-Ghamdi M, Al-Ayadhi L, El-Ansary A. Selected biomarkers as predictive tools in testing efficacy of melatonin and coenzyme $\mathrm{Q}$ on propionic acid - induced neurotoxicity in rodent model of autism. BMC Neurosci, 2014; 15:34.

39. Yuan Fan Yang YF, Lai XY, Yin Lai GY, Ze Dong JZD, Ni H, Chen F. Purification and characterization of a tyrosinase inhibitor from camellia pollen. J Funct Foods. 2016:27:140-9.

40. Kim SR, Park MJ, Lee MK, Sung SH, Park EJ, Kim J, Kim SY, Oh TH, Markelonis GJ, Kim YC. Flavonoids of Inula britannica protect cultured cortical cells from necrotic cell deathinduced by glutamate. Free Radic Biol Med. 2002;32(7): 596-604.

41. Franco $\mathrm{L}$, Thais Posser Fabiana Missau, Pizzolatti MG, dos Santos ARS, Souza DO, Aschner M, Rocha JBT, Dafre AL, Farina M. Structure-activity relationship of flavonoids derived from medicinal plants in preventing methylmercury-induced mitochondrial dysfunction. Environ Toxicol Pharmacol. 2010;30(3):272-8.

\section{Submit your next manuscript to BioMed Central and we will help you at every step:}

- We accept pre-submission inquiries

- Our selector tool helps you to find the most relevant journal

- We provide round the clock customer support

- Convenient online submission

- Thorough peer review

- Inclusion in PubMed and all major indexing services

- Maximum visibility for your research

Submit your manuscript at www biomedcentral.com/submit
C BioMed Central 